\title{
Imaging Features of Malignant Lacrimal Sac and Nasolacrimal Duct Tumors
}

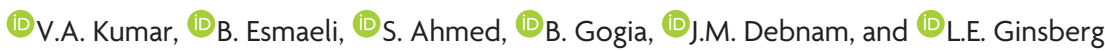

\begin{abstract}
SUMMARY: The purpose of this study was to present the imaging features of primary and secondary malignant lacrimal sac and nasolacrimal duct tumors and their pattern of tumor spread in 18 patients. The most common tumor histology in our series was squamous cell carcinoma. In 15/18 patients, tumor involved both the lacrimal sac and duct at the time of diagnosis. In 11/16 patients on CT, the nasolacrimal bony canal was smoothly expanded without erosive changes. The medial canthus region (16/18) was a frequent site of direct tumor spread. Two patients had intraconal orbital spread of tumor. Tumor spread to the sinus or nasal cavity was observed in 5/13 primary tumors. Only 1 patient presented with nodal metastasis. There was no intracranial tumor extension, perineural tumor spread along the infraorbital nerve, distant metastasis, or dacryocystocele formation in any of the patients at the time of diagnosis.
\end{abstract}

ABBREVIATION: SCCA = squamous cell carcinoma

$\mathbf{T}$ he lacrimal drainage apparatus consists of the superior and inferior canaliculi, common canaliculus, lacrimal sac, and nasolacrimal duct (Fig 1). ${ }^{1}$ Tears collect in the lacrimal sac before draining into the nasolacrimal duct and the inferior meatus of the nasal cavity. ${ }^{2}$ Lacrimal sac and duct tumors are rare. ${ }^{3}$ The most common presenting clinical symptoms of lacrimal sac and duct tumors are epiphora, recurrent dacryocystitis, epistaxis, and/or a lacrimal sac mass. ${ }^{3}$ These tumors present with nonspecific symptoms suggestive of chronic dacryocystitis; often diagnosis and treatment are delayed. ${ }^{4}$ Early diagnosis and treatment are often curative and can prevent tumor recurrence and loss of the eye and visual function. ${ }^{4,5}$ The tumors are divided into epithelial and nonepithelial neoplasms. Benign epithelial tumors include squamous and transitional cell papillomas and oncocytomas. The malignant epithelial neoplasms include squamous cell carcinoma (SCCA), transitional cell carcinoma, adenocarcinoma, adenoid cystic carcinoma, poorly differentiated carcinoma, and, rarely, primary melanomas. ${ }^{5}$ Secondary involvement of the lacrimal sac and duct can occur from any cutaneous lesion that involves the eyelid and/or conjunctiva, including SCCA, basal cell carcinoma, and sebaceous cell carcinoma, and from any neoplastic process in-

Received February 10, 2016; accepted after revision June 2.

From the Department of Diagnostic Radiology (V.A.K., S.A., B.G., J.M.D, L.E.G.), and Orbital Oncology and Ophthalmic Plastic Surgery Program, Department of Plastic Surgery (B.E.), University of Texas MD Anderson Cancer Center, Houston, Texas.

Please address correspondence to Vinodh A. Kumar, MD, Department of Diagnostic Radiology, Unit 1482, University of Texas MD Anderson Cancer Center, 1400 Pressler St, Houston, TX 77030; e-mail: vakumar@mdanderson.org

http://dx.doi.org/10.3174/ajnr.A4882 volving the paranasal sinuses. Metastatic lesions to the lacrimal sac can originate from any distant site and may include carcinomas or melanomas.

The purpose of this study was to review the CT and MR imaging features of malignant lacrimal sac and nasolacrimal duct tumors. We investigated the extent of tumor involvement of the lacrimal sac and duct and the extent of bony expansion or destruction of the nasolacrimal duct bony canal. We characterized the malignant lacrimal sac and duct tumors by the degree of enhancement, signal characteristics on MR imaging, and local/regional spread. We also sought to determine whether dacryocystocele formation was a feature associated with these tumors and whether nodal metastasis, distant metastasis, perineural tumor spread along the infraorbital nerve, or intracranial tumor extension was present on imaging at the time of the initial evaluation.

\section{MATERIALS AND METHODS \\ Case Series}

Approval for this retrospective Health Insurance Portability and Accountability Act-compliant study was obtained from the institutional review board, with a waiver of informed consent. A retrospective analysis was performed on 18 patients, 11 women and 7 men, 45-86 years of age (mean, 64 years), who had been treated for malignant lacrimal sac and nasolacrimal duct tumors at our institution. Thirteen patients had primary malignant tumors of the lacrimal sac and/or nasolacrimal duct, 4 patients had contiguous malignant tumor spread to the lacrimal sac and/or duct from a neighboring site, and 1 patient had a metastatic melanoma to the lacrimal sac and duct. The tumor histology consisted of the 


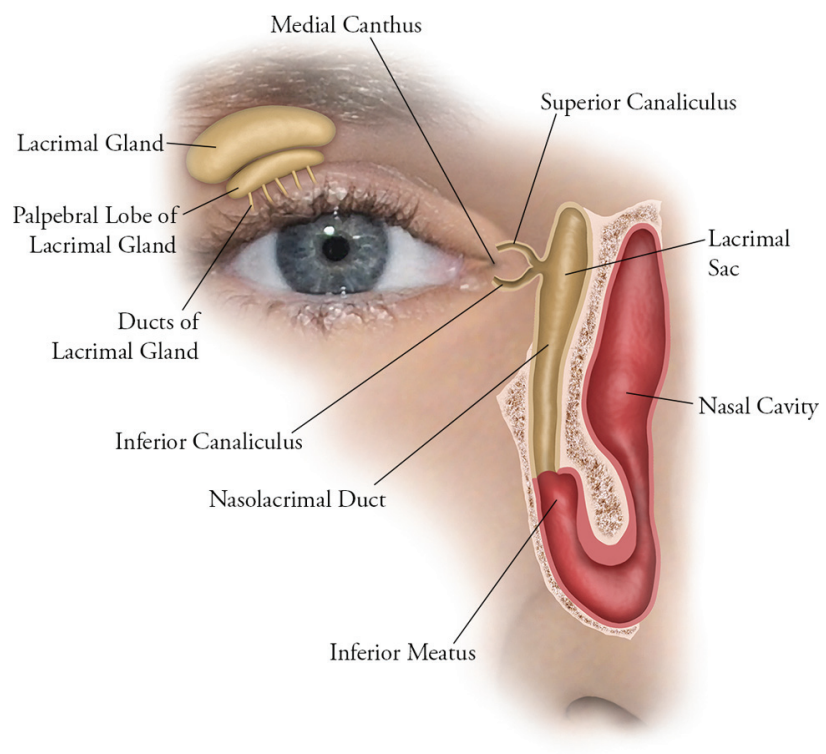

FIG 1. Normal anatomy of the lacrimal drainage system apparatus, which includes the canaliculi, lacrimal sac, and nasolacrimal duct.

following: SCCA $(n=7)$, poorly differentiated sebaceous carcinomas $(n=3)$, melanomas $(n=3)$, adenocarcinomas $(n=2)$, adenoid cystic carcinoma $(n=1)$, small cell undifferentiated carcinoma $(n=1)$, and papillary transitional carcinoma $(n=1)$.

CT was performed on HD 750 scanners (GE Healthcare, Milwaukee, Wisconsin) after the administration of intravenous contrast by using the following parameters: $120-140 \mathrm{kV}$ (peak); $180-$ $220 \mathrm{~mA}$; section thickness, $1.25 \mathrm{~mm}$; FOV, 180 to 250 . MR imaging studies were performed on 1.5T MR imaging scanners (Excite HDxt; GE Healthcare) with the following pulse sequences: T1WI, T2WI, and postcontrast T1WI. The images were reviewed by 2 head and neck radiologists (V.A.K. and L.E.G.).

The degree of contrast enhancement of the malignant lacrimal sac and nasolacrimal duct tumors on CT and MR imaging was graded relative to the degree of contrast enhancement of the normal lacrimal glands. For this study, a normal lacrimal gland demonstrated moderate enhancement on postcontrast CT and intense contrast enhancement on postcontrast T1WI.

\section{RESULTS}

The results of this study are summarized in the Table. In 15/18 patients, malignant tumor involved both the lacrimal sac and nasolacrimal duct at the time of diagnosis. Only 3 patients had tumor confined to the lacrimal sac. Tumor was not observed solely within the nasolacrimal duct in any patient. In 16/18 patients, tumor had spread from the lacrimal sac into the medial canthus region (Figs 2 and 3). Two patients had intraconal orbital tumor spread (Fig 4A).

In $11 / 16$ patients who had undergone CT, the nasolacrimal bony canal was smoothly expanded without erosive changes (Fig $5 B)$. In 2 patients, there were erosive changes to the nasolacrimal duct bony canal. One patient with possible erosive changes had undergone a prior dacryocystorhinostomy; therefore, the findings were iatrogenic. In 2 patients, there was no nasolacrimal duct dilation or erosion because these tumors were diagnosed at an early stage.
Imaging features and regional spread of malignant lacrimal sac and nasolacrimal duct tumors

\begin{tabular}{|c|c|}
\hline Features & $\begin{array}{l}\text { No. of } \\
\text { Patients }\end{array}$ \\
\hline \multicolumn{2}{|l|}{$\begin{array}{l}\text { Tumor location along lacrimal system on CT } \\
\text { and MRI }(n=18)\end{array}$} \\
\hline Lacrimal sac only & 3 \\
\hline Nasolacrimal duct only & 0 \\
\hline Involving lacrimal sac and nasolacrimal duct & 15 \\
\hline \multicolumn{2}{|l|}{$\begin{array}{l}\text { Tumor involvement of the nasolacrimal duct bony } \\
\text { canal on CT }(n=16)\end{array}$} \\
\hline No duct dilation & 2 \\
\hline Smoothly expanded duct & 11 \\
\hline Erosive/lytic changes to duct & 2 \\
\hline $\begin{array}{l}\text { latrogenic changes to duct from prior } \\
\text { dacryocystorhinostomy }\end{array}$ & 1 \\
\hline \multicolumn{2}{|l|}{ Orbit involvement by tumor $(n=18)$} \\
\hline Medial cantus/extraconal space of orbit & 16 \\
\hline Intraconal space of orbit & 2 \\
\hline \multicolumn{2}{|l|}{$\begin{array}{l}\text { Sinonasal involvement by tumor in primary malignant } \\
\text { tumors of the lacrimal sac or duct }(n=5)^{\mathrm{a}}\end{array}$} \\
\hline Ethmoid sinus & 4 \\
\hline Maxillary sinus & 3 \\
\hline Nasal cavity & 5 \\
\hline \multicolumn{2}{|l|}{ Other findings $(n=18)$} \\
\hline Nodal metastasis & 1 \\
\hline Distant metastasis & 0 \\
\hline Perineural tumor spread along the infraorbital nerve & 0 \\
\hline Intracranial extension & 0 \\
\hline Dacryocystocele formation & 0 \\
\hline
\end{tabular}

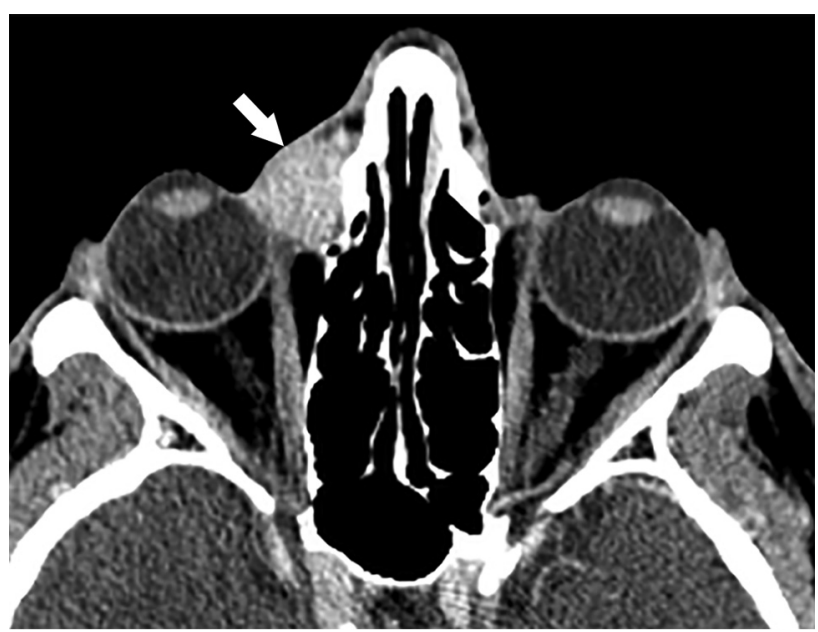

FIG 2. A 46-year-old woman with moderately differentiated invasive SCCA of the right lacrimal sac and nasolacrimal duct. Post-contrast-enhanced CT shows tumor extension from the lacrimal sac into the medial canthus region (arrow), which is a common site of tumor spread.

Of the 16 cases of malignant lacrimal sac and duct tumors evaluated with CT, 3 demonstrated mild contrast enhancement, 9 showed moderate contrast enhancement, and 4 exhibited intense enhancement. In the 12/13 patients who had undergone MR imaging, the lacrimal sac and duct tumors were isointense on T1WI, with the exception of 1 patient with nasal melanoma, which demonstrated high intensity on T1WI. In all 13 patients, the tumors showed isointense signal on T2WI (Fig 3B). On MR imaging, 3 cases demonstrated mild contrast enhancement, 7 cases had moderate contrast enhancement, and 3 exhibited intense contrast enhancement.

AJNR Am J Neuroradiol 37:2134-37 Nov 2016 www.ajnr.org 2135 

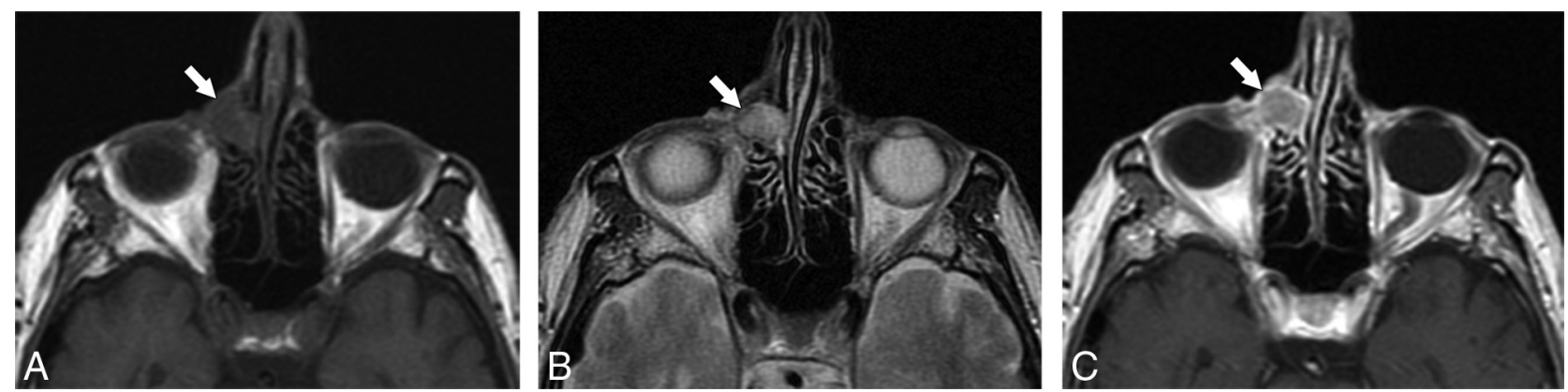

FIG 3. An 85-year-old man with metastatic melanoma to the lacrimal sac and nasolacrimal duct with extension into the medial canthus region. Axial MR imaging demonstrates an isointense mass in the right medial canthus on TIWI $(A)$, isointensity on T2WI (B), and enhancement on postcontrast TIWI $(C)$
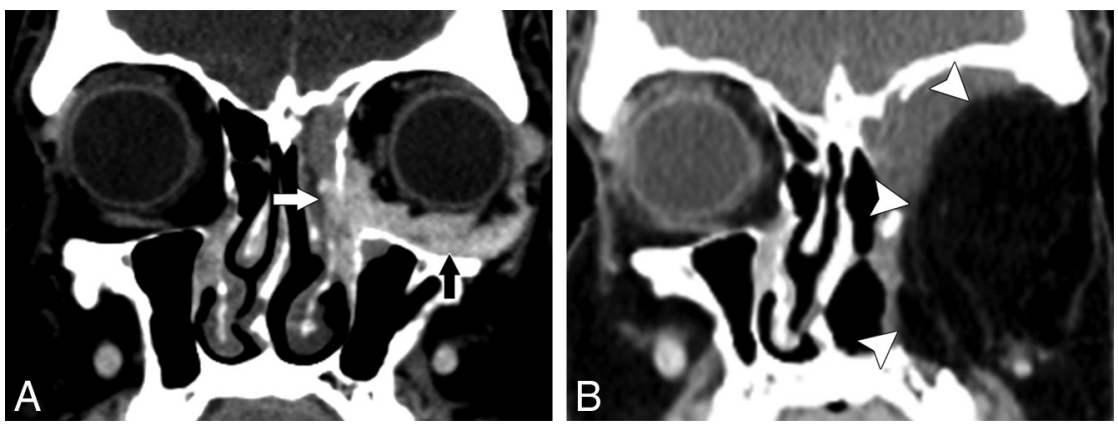

\section{DISCUSSION}

To our knowledge, no large imaging series has assessed malignant lacrimal sac and nasolacrimal duct tumors. This report highlights the CT and MR imaging features and spread pattern of these tumors. In our series, the most common tumor histology was SCCA (39\%), which has also been reported in other studies. ${ }^{5,6}$ Eighty-three percent of primary and secondary tumors in our study

FIG 4. A 50-year-old man with adenocarcinoma of the left lacrimal sac and nasolacrimal duct. $A$, Postcontrast thin-section coronal CT reformatted images demonstrate tumor in the lacrimal sac and duct (white arrow) with direct extension into the inferior intraconal orbital space (black arrow). B, Due to the extent of tumor spread, the patient underwent left orbital exenteration, maxillectomy, and free flap reconstruction (white arrowheads) with adjuvant radiation therapy and has been disease-free for 3 years.
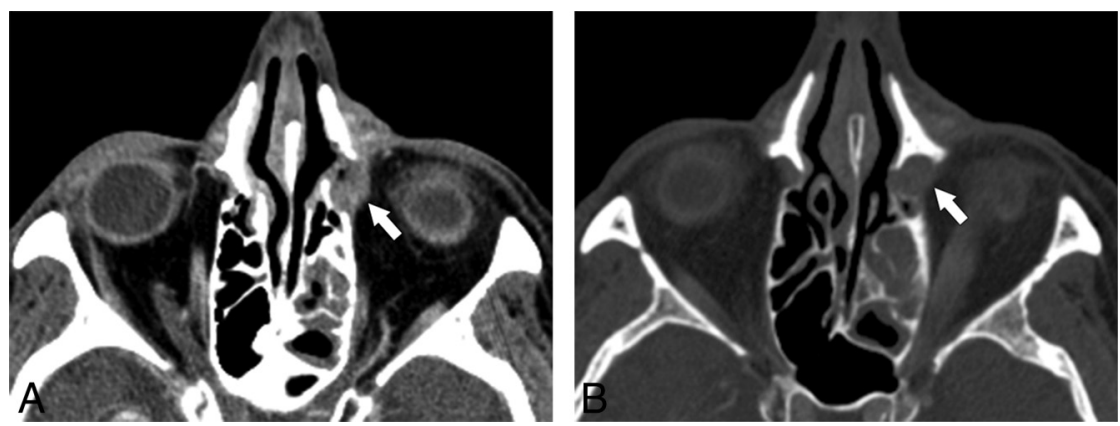

FIG 5. A 73-year-old woman with well-differentiated SCCA of the lacrimal sac and nasolacrimal duct. $A$, Post-contrast-enhanced CT demonstrates an enhancing tumor within the left lacrimal sac (arrow). B, At a slightly more inferior level (bone window), note the mild expansion of the lacrimal bony canal by tumor (arrow). involved both the lacrimal sac and nasolacrimal duct at the time of initial imaging. The medial canthus region (Figs 2 and 3) was commonly involved by malignant tumors (89\%) because of direct tumor spread from the lacrimal sac; intraconal orbital extension of tumor was less common (11\%). The differential diagnosis of masses in the medial canthus includes lacrimal sac and duct neoplasms, but more commonly, dacryocystitis or idiopathic nasolacrimal duct blockage is a cause. $^{5}$ In our series, $38 \%$ of the primary malignant nasolacrimal sac or nasolacrimal duct tumors demonstrated spread to either the sinus or nasal cavity.

Tumor abutted the infraorbital foramen in 2 patients with advanced disease. However, perineural tumor spread along the infraorbital nerve was not seen on imaging or on histology. Intracranial tu-

Five of 13 patients with primary malignant lacrimal sac and duct tumors had disease spread to the sinus or nasal cavity. Four had ethmoid sinus tumor extension, and 3 had maxillary sinus tumor involvement. Five had nasal cavity tumor extension, with 4 specifically involving the inferior meatus of the nasal cavity. Note that some patients had $>1$ subsite of sinonasal tumor extension. Postobstructive sinus secretions were noted in 3 patients.

One patient presented with an ipsilateral jugulodiagastric (level IIA) nodal metastasis at the time of initial diagnosis. Dacryocystocele formation, perineural tumor spread along the infraorbital nerve, intracranial extension of tumor or distant metastases were not seen in any of the patients at the time of initial diagnosis. mor extension or distant metastases were not observed in any patient at the time of diagnosis. Nodal metastasis was seen in only 1 patient in our series. In a large ophthalmology study, $\mathrm{Ni}_{\mathrm{et} \mathrm{al}^{7}}^{7}$ found distant metastases from malignant lacrimal sac tumors in only $6 / 74$ patients and intracranial tumor spread in $1 / 74$ patients. They found nodal metastasis in $28 \%$ of patients, generally months to years after the initial diagnosis. ${ }^{7}$ When present, nodal metastases from malignant lacrimal sac and duct tumors generally involve the preauricular, submandibular, and cervical lymph nodes. ${ }^{7,8}$

In $69 \%$ our cases imaged with CT, the malignant lacrimal sac and duct tumors expanded the lacrimal bony canal (Fig 5B) without erosion. CT was superior to MR imaging in the delineation of 
the lacrimal bony canal and in evaluating expansion versus erosion of the canal. Thin-section coronal imaging was very helpful on both CT and MR imaging in the interpretation of the study because it nicely displayed the entirety of the lacrimal sac and duct and regional tumor spread (Fig $4 A$ ). When postobstructive sinus secretions were present ( 3 cases), MR imaging was superior to CT in distinguishing tumor from secretions within the sinonasal cavity. In 2 cases, MR imaging and CT were equivalent in the diagnostic evaluation of intraconal tumor spread. We found that the lacrimal canaliculi were not easily identifiable on MR imaging and CT, due to the small size of these anatomic structures. In cases of locally advanced malignant lacrimal sac and duct tumors, we found it difficult to distinguish primary tumors from secondary tumors by imaging. We anticipated seeing dacryocystocele formation secondary to tumor obstruction of the nasolacrimal duct more frequently; however, this was not seen in any case.

In most cases in this study, the malignant lacrimal sac and nasolacrimal sac tumors demonstrated moderate contrast enhancement on CT and MR imaging and demonstrated isointense signal intensity on T1-weighted and T2-weighted MR imaging. Contrast enhancement and MR imaging signal intensity were not helpful in distinguishing tumor histology, except in 1 case of recurrent nasal cavity melanoma, which spread to the nasolacrimal duct and demonstrated high T1WI signal intensity. Primary malignant melanoma of the lacrimal sac is rare, accounting for only $5 \%$ of lacrimal sac tumors. ${ }^{9}$ Due to the paramagnetic properties of melanin, melanoma may appear hyperintense on T1WI and hypointense on T2WI. ${ }^{6}$ According to Billing et al, ${ }^{9}$ melanoma may also demonstrate isointense T1WI and T2WI signal on MR imaging (Fig $3 A,-B)$.

Treatment of primary malignant lacrimal sac and nasolacrimal duct tumors typically involves wide local resection, followed by radiation and/or chemotherapy. In our series, surgical excision of the lacrimal sac was complemented by a medial maxillectomy to allow complete resection of the nasolacrimal duct. Judicious use of postoperative adjuvant proton radiation therapy or concurrent chemoradiation therapy can yield good local/regional control, and a globe-sparing surgery can be achieved in most cases with reasonable visual outcomes. ${ }^{5}$ Bony erosion of the nasolacrimal bony canal does not portend a worse prognosis, but it may dictate the size of the planned bony resection, which, in addition to a medial maxillectomy, may also include an ethmoidectomy. Larger tumor diameter (generally $>30 \mathrm{~mm}$ ) at the time of presentation $^{5}$ and tumor invasion of the intraconal space of the orbit may necessitate orbital exenteration (Fig 4A, -B).

Imaging surveillance of the head and neck is recommended every 3 months for the first year, every 6 months the second year, and annually in the third year and beyond. ${ }^{5}$ On the basis of the results of this study, the authors recommend performing a thinsection $(1.25 \mathrm{~mm}) \mathrm{CT}$ with contrast as the first-line imaging study to evaluate malignant lacrimal sac and nasolacrimal duct tumors at the time of initial staging. MR imaging can be performed if CT cannot distinguish sinonasal tumor extension from postobstructive secretions.

\section{CONCLUSIONS}

Malignant lacrimal sac and nasolacrimal duct tumors tend to expand the nasolacrimal bony canal, rather than erode it. CT was superior to MR imaging in characterizing expansion versus erosion of the nasolacrimal bony canal. In most cases, tumor involved both the lacrimal sac and nasolacrimal duct at initial diagnosis. The medial canthus region was a frequent site of tumor spread. Tumor spread to the sinus or nasal cavity was observed in less than half of the primary tumor cases. Nodal metastasis was seen in only 1 patient. There was no intracranial tumor extension, perineural tumor spread along the infraorbital nerve, distant metastasis, or dacryocystocele formation in any of the cases at the time of initial imaging. The most common tumor histology in our series was SCAA.

Disclosures: Bita Esmaeli-UNRELATED: Other: Roche; I served on their Data Safety Monitoring Board last year.

\section{REFERENCES}

1. Debnam JM, Esmaeli B, Ginsberg LE. Imaging characteristics of dacryocystocele diagnosed after surgery for sinonasal cancer. $A J N R$ Am J Neuroradiol 2007;28:1872-75 CrossRef Medline

2. Russell EJ, Czervionke L, Huckman M, et al. CT of inferomedial orbit and lacrimal drainage apparatus: normal and pathologic anatomy. AJR Am J Roentgenol 1985;145:1147-54 CrossRef Medline

3. Stefanyszyn MA, Hidayat AA, Pe'er JJ, et al. Lacrimal sac tumors. Ophthal Plast Reconstr Surg 1994;10:169-84 CrossRef Medline

4. De Stefani A, Lerda W, Usai A, et al. Squamous cell carcinoma of lacrimal drainage system: case report and literature review. Tumori 1998;84:506-10 Medline

5. El-Sawy T, Frank SJ, Hanna E, et al. Multidisciplinary management of lacrimal sac/nasolacrimal duct carcinomas. Ophthal Plast Reconstr Surg 2013;29:454-57 CrossRef Medline

6. Weber AL, Rodriguez-DeVelasquez A, Lucarelli MJ, et al. Normal anatomy and lesions of lacrimal sac and duct: evaluated by dacryocystography, computed tomography, and MR imaging. Neuroimaging Clin N Am 1996;6:199-217 Medline

7. Ni C, D'Amico DJ, Fan CQ, et al. Tumors of lacrimal sac: a clinicopathological analysis of 82 cases. Int Ophthalmol Clin 1982;22: 121-40 CrossRef Medline

8. Rahangdale SR, Castillo M, Shockley W. MR in squamous cell carcinoma of the lacrimal sac. AJNR Am J Neuroradiol 1995;16:1262-64 Medline

9. Billing K, Malhotra R, Selva D, et al. Magnetic resonance imaging findings in malignant melanoma of the lacrimal sac. $\mathrm{Br} J$ Ophthalmol 2003;87:1187-88 CrossRef Medline 